\title{
A "Swimming" Heart
}

Marcello Di Valentino ${ }^{1^{*}}$, Vito Spataro ${ }^{2}$ and Andreas Perren ${ }^{3}$

${ }^{1}$ Department of Cardiology, Ospedale San Giovanni, 6500 Bellinzona, Switzerland

${ }^{2}$ Oncology Institute of Southern Switzerland, 6500 Bellinzona, Switzerland

3Intensive Care Unit, Ospedale San Giovanni, 6500 Bellinzona, Switzerland

*Corresponding author: Marcello Di Valentino, Department of Cardiology, Ospedale San Giovanni, CH - 6500 Bellinzona, Switzerland, Tel: +41 9181181 38; E-mail: marcello.divalentino@eoc.ch

Received date: June 26, 2015; Accepted date: July 13, 2015; Published date: July 15, 2015

Copyright: ( 2015 Di Valentino M. This is an open-access article distributed under the terms of the Creative Commons Attribution License, which permits unrestricted use, distribution, and reproduction in any medium, provided the original author and source are credited.

\section{Case}

A 61-year-old man was seen by an oncologist for evaluation of a pluri-metastatic pulmonary adenocarcinoma. Thoracic computed tomography, performed two weeks earlier (Figure 1A) showed a leftsided pleural effusion and pericardial metastasis with a small pericardial effusion. During physical examination the patient presented shortness of breath and dizziness. Jugular venous distension, sinus tachycardia $(140 / \mathrm{min})$ and pronounced systolic arterial hypotension $(70 \mathrm{mmHg}$ ) with pulsus paradoxus prompted the suspicion of cardiac tamponade.

In the emergency room a transthoracic echocardiography subcostal view revealed a "swimming" heart with swinging motion due to a huge, relevant pericardial effusion producing diastolic collapse of the free right ventricular wall (Figure 1B).

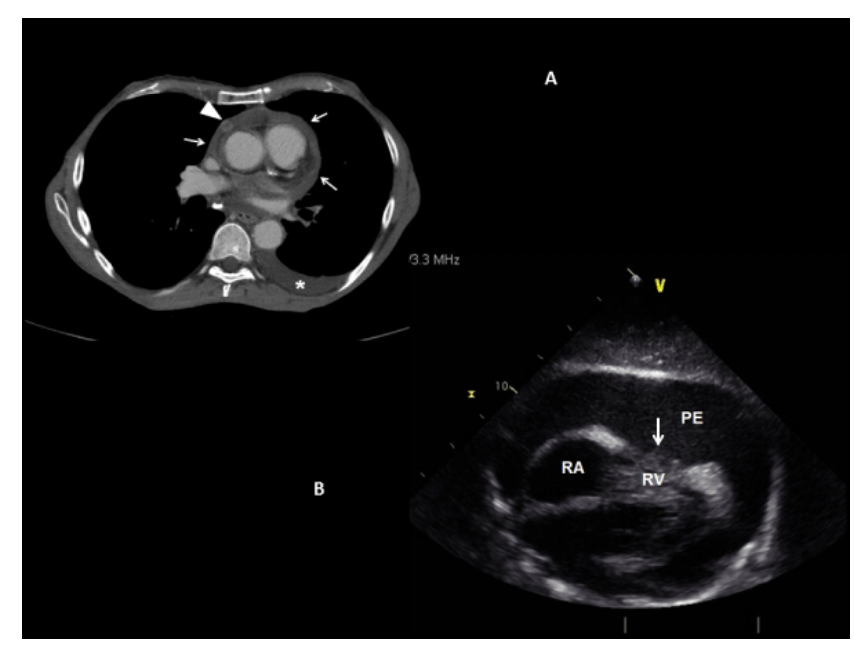

Figure 1: (A) Thoracic CT scan, showed a left-sided pleuric effusion $\left.{ }^{*}\right)$, pericardic metastasis (arrowhead) and small pericardial effusion (arrows); (B) Transthoracic echocardiogram (subcostal 4 chamber view) revealed a large pericardial effusion (PE) with diastolic collapse of the free right ventricular wall (head, RV). RA= right atrium; $\mathrm{RV}=$ right ventricle; $\mathrm{PE}=$ pericardial effusion

An urgent needle pericardiocentesis was performed and just $200 \mathrm{ml}$ of sero-hemorrhagic fluid were removed, and signs and symptoms of cardiac tamponade rapidly disappeared. A total of $1200 \mathrm{ml}$ of liquid was progressively evacuated. Repeat echocardiogram revealed no relapse of pericardial effusion, a quasi-voided pericardial space and good diastolic distension of the free right ventricular wall (Figure 2). Consecutively, a pericardiodesis whith cytomicin was affected.

Cardiac tamponade is the accumulation, acute or sub-acute, of pericardial fluid leading to compression of all cardiac chambers due to increased pericardial pressure [1]. Metastatic lung cancer is one of the causes which induce a pericardial hemorrhagic effusion [2].

Although clinical history and physical examination are important elements to suspect cardiac tamponade, the two-dimensional and Doppler echocardiography play major roles in the identification of pericardial effusion and in assessing its hemodynamic significance [3]. In the present case the diagnosis was reached through the interpretation of clinical history and of the clinical signs. The initial suspicion of cardiac tamponade was confirmed by echocardiography, which demonstrates a "swimming" heart into a large pericardial effusion suggesting hemodynamic compromise of the right chamber.

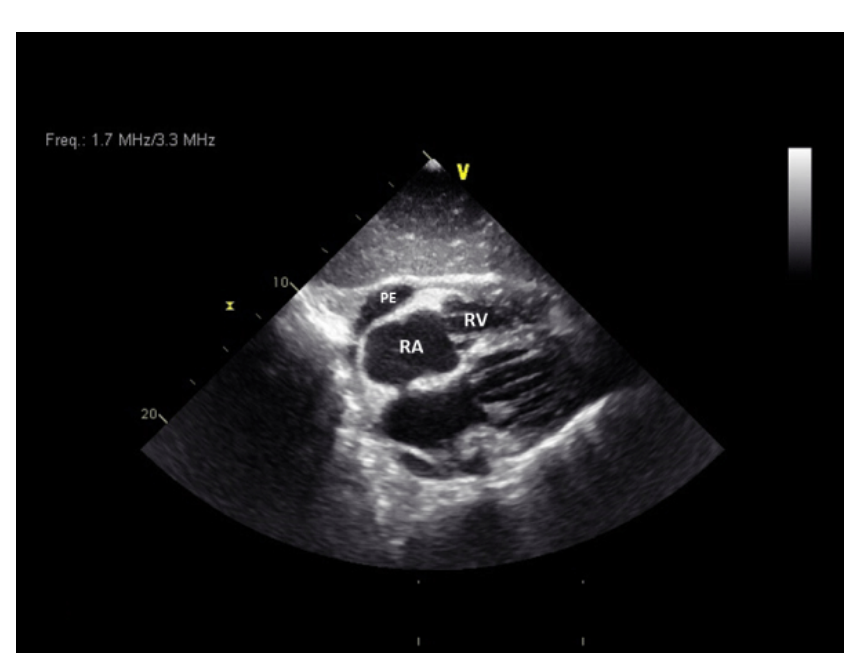

Figure 2: Transthoracic echocardiogram (subcostal 4 chamber view) show minor pericardial effusion (PE) without diastolic collapse of the free right ventricular wall $(\mathrm{RV}) . \mathrm{RA}=$ right atrium; $\mathrm{RV}=$ right ventricle; $\mathrm{PE}=$ pericardial effusion

\section{References}

1. Spodick DH (2003) Acute cardiac tamponade. N Engl J Med 349: 684-690.

2. Permanyer-Miralda G (2004) Acute pericardial disease: approach to the aetiologic diagnosis. Heart 90: 252-254. 
Citation: Valentino MD, Spataro V, Perren A (2015) A “Swimming” Heart. J Cardiovasc Dis Diagn 3: 208. doi:10.4172/2329-9517.1000208

Page 2 of 2

3. Imazio M, Adler Y (2013) Management of pericardial effusion. Eur Heart J 34: 1186-1197. 